\title{
FOOTWEAR CHARACTERISTICS AND FACTORS INFLUENCING FOOTWEAR CHOICE IN PATIENTS WITH GOUT
}

Professor Keith Rome (PhD) ${ }^{1}$, Mike Frecklington (BSc (Hons) Pod) ${ }^{1}$, Professor Peter McNair (PhD) ${ }^{1}$, Associate Professor Peter Gow (FRACP, MBChB, BMedSci) ${ }^{4}$, Associate Professor Nicola Dalbeth (MBChB, MD, FRACP) ${ }^{2,3,4}$

${ }^{1}$ AUT University, Health \& Rehabilitation Research Institute, Auckland, New Zealand,

${ }^{2}$ Counties Manukau District Health Board, Auckland, New Zealand

${ }^{3}$ Auckland District Health Board, Auckland, New Zealand,

${ }^{4}$ University of Auckland, Auckland, New Zealand.

Corresponding author:

Professor Keith Rome

Division of Rehabilitation \& Occupation Studies,

AUT University, Akoranga Drive,

Private Bag 92006, Auckland 1020, New Zealand.

Tel: $+649921-9999$ ext 7688

Fax: +6499219839

email: krome@aut.ac.nz

Word count: 2258

Financial Support: Mike Frecklington - AUT Summer Studentship 


\section{ABSTRACT}

Objective. Gout is associated with foot pain, impairment and disability. The aim of this study was to assess footwear characteristics and key factors influencing footwear choice in patients with gout. We also wished to evaluate the relationship between footwear characteristics and foot disability.

Methods. Fifty patients with a history of acute gout were recruited from rheumatology clinics during the summer months. Clinical characteristics, global function, and foot impairment and disability measures were recorded. Footwear characteristics and the factors associated with choice of footwear were identified using validated assessment tools. Suitability of footwear was assessed using pre-determined criteria for assessing adequacy of footwear, based on a previous study of foot pain.

Results. The patients had moderate to severe foot pain, impairment and disability. Poor footwear characteristics included poor cushioning, lack of support, lack of stability and motion control. Over $50 \%$ of shoes were $\geq 12$ months old and demonstrated excessive wear patterns. Patients reported comfort (98\%), fit (90\%), support (79\%) and cost (60\%) as important factors in choosing their own footwear. No correlation was found between footwear characteristics (length and width) and foot characteristics (foot pain, impairment and disability). Patients with poor footwear reported higher foot-related impairment and disability.

Conclusion. Use of poor footwear is common in patients with chronic gout and is associated with foot disability and impairment.

Key words: gout, footwear, foot, pain, disability

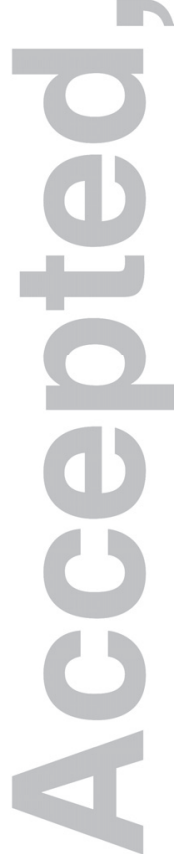




\section{Significance and Innovations}

- Poor footwear is a major problem in patients with gout

- Foot pain, impairment and disability may contribute to the problem.

- Patients reported comfort, fit, support and cost as important factors in choosing their own footwear.

- Future research should be focused on assessing the role of competitively priced footwear with adequate cushioning, motion control and sufficient width at the forefoot. 


\section{INTRODUCTION}

Gout is the most prevalent inflammatory arthropathy in men, caused by formation of monosodium urate crystals in joints and other tissues (1). Gout typically presents as recurrent self-limiting flares of acute joint inflammation, and in the presence of persistent hyperuricaemia, chronic tophaceous disease may also develop (2). Gout displays a striking predilection to affect the feet, particularly the first metatarsophalangeal joint (1st MTPJ), midfoot and ankle (3-6). We have recently reported that patients with chronic gout have changes in gait parameters focused on the midfoot and the hallux, consistent with a pain avoidance strategy (7). It is likely that these gait changes contribute to altered loading patterns and impaired foot function in chronic gout.

Footwear has been developed and modified to provide protection from the environment, conform to fashion, assist function, accommodate foot deformities, and treat musculoskeletal injury (8). Various footwear characteristics have been linked to the development of musculoskeletal disorders such as osteoarthritis of the foot and knee, low back pain, foot ulceration, hallux valgus and hammer toes (8). Poorly fitting shoes have also been linked to foot pain in rheumatoid arthritis $(9,10)$. In addition to shoe features, previous studies have reported that the individual fit of a shoe is important; loose-fitting shoes can also cause excessive foot slippage within the shoe during walking, altered contact area between the foot and shoe, impairing foot stability and walking parameters (11-13).

To date, the choice of footwear and factors impacting on the choice of footwear have been not been reported in patients with chronic gout. The aim of this study was to assess footwear characteristics and key factors influencing footwear choice in patients with gout. We also wished to examine the relationships between footwear and foot characteristics (pain, disability and impairment).

\section{PATIENTS AND METHODS}

This was a cross-sectional observational study of fifty adult patients with a history of gout attacks recruited from rheumatology outpatient clinics at Auckland and Counties 
Manukau District Health Boards, Auckland, New Zealand. All patients had a physician diagnosis of gout and a history of acute gout according to ACR classification criteria (14). Ethical approval was obtained by Northern $X$ Ethics Committee, Auckland, New Zealand (NTX/10/EXP/231) and local institutional approval was also obtained. Participants were excluded if they were experiencing an acute gout flare at the time of assessment or had lower limb amputation. Patients with diabetes or neurological disease associated with gout were not excluded from the study. A single podiatrist (MF) assessed all patients at a single study visit.

The following data were collected: age, gender, ethnicity, body mass index (BMI), disease duration, current pharmacological management, and history of cardiovascular disease and diabetes. Foot type was assessed using the Foot Posture Index which is a validated method for quantifying standing foot type with scores above +4 suggest a flat-foot type (15).

Disease impact was measured using the Leeds Foot Impact Scale (16). This selfadministered questionnaire comprises two subscales for impairment/footwear (LFIS $\mathrm{IF}_{\mathrm{F}}$ ) and activity limitation/participation restriction (LFIS $\left.\mathrm{AP}\right)$. The former contains 21 items related to foot pain and joint stiffness as well as footwear related impairments and the latter contains 30 items related to activity limitation and participation restriction. Turner (17) reports that a $\mathrm{LFIS}_{\mathrm{IF}}>7$ point and $\mathrm{LFIS}_{\mathrm{AP}}>10$ point as a high to severe level of foot impairment and disability.

Foot pain was assessed using the Foot Function Index domain (17). The FFI is a self-administered questionnaire consisting of 23 items grouped in three domains: foot pain (nine items), disability (nine items) and functional limitation (five items). All items are rated using $100 \mathrm{~mm}$ visual analogue scales, and higher scores indicate greater pain, disability and limitation of activity and thus poorer foot health (18).

An objective assessment of footwear was conducted by the examiner to ascertain the type, structural components and fit of the participant's footwear at the time of the study visit (8). Patients did not receive any instructions about their footwear prior to the study visit. Six aspects of footwear are evaluated and include: (i) fit (length, 
width and depth); (ii) general (age of shoe, footwear style, weight and length); (iii) general structure (heel height, fixation, forefoot height, forefoot sole flexion point, and last); (iv) motion control properties (density, fixation, heel counter stiffness, midfoot sole sagittal and frontal stability); (v) cushioning (presence lateral, medial and heel sole hardness); (vi) wear patterns (upper, midsole, tread and outsole wear pattern).

Based upon previous studies of patients with foot pain and rheumatoid arthritis, we classified current footwear into poor, average and good footwear $(10,20)$. The poor footwear group consisted of footwear that lack support and sound structure, including sandals, flip-flops, slippers, mules and moccasins. The average footwear group included shoes such as hard-or-rubber-soled shoes and work boots. The good footwear group consisted of athletic shoes, walking shoes, therapeutic footwear and Oxford-type shoes. Foot dimensions (foot length and width) were measured using a Brannock measuring device (Liverpool, New York, USA). The device allows the weight-bearing measurement of foot length and width. Each patient was also asked by the examiner to identify the most important feature on a validated check-list that included: comfort, style, fit, sole, costs, weight and colour (21).

All analyses were performed using SPSS V17.0. Gender, ethnicity, clinical characteristics such as current pharmacological management, history of hypertension, cardiovascular disease, diabetes, renal impairment and general footwear scores are described as $\mathrm{n}$ (percentages). All other demographic characteristics are described as the mean (SD). The association of changes with foot characteristics (pain, disability and impairment) with footwear characteristics (shoe length and width) were evaluated using Spearman's $\rho$ correlation coefficients. We examined significant differences between shoe category (good, poor and average) and foot characteristics (pain, disability and impairment) using one-way ANOVA. We undertook secondary analysis using independent t-tests to evaluate significant differences in all footwear characteristics between participants with diabetes and those without diabetes. All tests were two tailed and $P<0.05$ was considered significant. 


\section{RESULTS}

Clinical and foot characteristics

The clinical and foot characteristics are summarised in Table 1. Patients were predominantly middle-aged males with longstanding disease. Obesity and cardiovascular disease were common comorbidities. The majority of patients $(n=27$, $58 \%$ ) had a low-foot profile (flatfoot). Patients had high to severe (LFIS IF $>7$ points $(n=27,52 \%), L_{\text {LIS }}>10$ points, $n=30,60 \%$ ) levels of foot impairment and disability (Table 1). The Foot Function Index, pain domain illustrated a moderate level of pain.

\section{Footwear assessment}

Table 2 summarises the footwear types observed. Overall, 28 (56\%) of patients wore good footwear that included walking, athletic and Oxford-type shoes, with 42\% of patients wearing shoes that were defined as 'poor'. No participants wore highheeled shoes.

Table 3 describes footwear characteristics. Footwear characteristics demonstrated that shoes were frequently either too long or too short. A similar finding was also found for shoe width, although shoe depth was deemed good in over $62 \%$ of patients. We found that $23 \%(n=12)$ of shoes had no fixation. Over $60 \%(n=30)$ of shoes demonstrated no cushioning and only $36 \%(n=18)$ of shoes with heel/forefoot cushioning. Minimal motion control properties was found in the current study with only $26 \%(n=13)$ wore shoes that had adequate heel counter stiffness, $50 \%(n=25)$ of shoes with midfoot sole sagittal stability and a further $42 \%(n=21)$ with midfoot sole frontal stability. We observed that $64 \%(n=32)$ of patients wore shoes with a heel height ranging between $2.6-5.0 \mathrm{~cm}$; of those $13(41 \%)$ wore athletic shoes, 9 (28\%) wore Oxford-type shoes, 7 (22\%) wore open shoes, $2(6 \%)$ wore bespoke footwear and 1 (3\%) wore boots. Forefoot sole flexion point demonstrated $54 \%$ $(n=27)$ of shoes before the level of the $1^{\text {st }}$ MPTJ. Over half of patients wore shoes that were aged over 12 months old.

Table 4 describes the factors patients perceived as important; most commonly identified factors were comfort $(98 \%)$, fit $(90 \%)$, support $(79 \%)$, cost $(60 \%)$ and 
weight (63\%). Patients reported style (36\%) and colour (33\%) as being less important.

Factors influencing changes in foot characteristics and footwear

Shoe width and depth did not correlate with foot pain, impairment and disability (data not shown). However, patients with poor footwear reported higher foot-related impairment and activities, particularly in the scores of the $\operatorname{LSIS}_{A P}(p=0.01)$ and the Foot Function Index, impairment domain ( $p=0.02)$ (Table 5). Secondary analysis demonstrated no significant differences in any of the footwear characteristics between participants with diabetes $(n=7)$ and those without diabetes $(n=43)(p>$ $\underline{0.05)}$.

\section{DISCUSSION}

The aim of this study was to identify current footwear styles, footwear characteristics, and factors that influence footwear choice experienced by patients with chronic gout. Overall, we found severe impairment and limited activity scores, consistent with significant foot disability and impairment associated with gout. A previous study has reported similar findings of foot pain, impairment and disability relating to chronic gout (7).

Over $40 \%$ of patients in the current study wore sandals, moccasins and flip-flops. A previous study (22) reported that gait changes were observed in an asymptomatic population with wearing flip-flops and suggested that the shoe construction may contribute to lower limb leg pain and are counter-productive to alleviating pain. The wearing of open-type footwear should be interpreted with caution in the current study. It is important to understand that open-type footwear, such as flip-flops and sandals are commonly worn in New Zealand. However, wearing open-type footwear may reflect the issue of finding appropriate footwear, in particular relating to finding footwear that has adequate foot width and length. 
Analysis of patient footwear illustrated signs of detrimental changes. Minimal motion control was found in the current study and since the midfoot is required to form a rigid lever during propulsion, footwear instability may contribute to foot problems in patients with chronic gout. The current study found over $50 \%$ of shoes with a flexion point distal to the level of the first metatarsophalangeal joint (1st MPJ). This may limit gait efficiency due to altered kinematics which results from inhibition of normal 1st MPJ function (8). We can postulate that a flexion point proximal may jeopardise the shoe's stability and may exacerbate the problem of efficient toe-off observed in patients with chronic gout (7).

Heel height greater than $2.5 \mathrm{~cm}$ has been associated with hallux valgus, plantar callus, postural instability in older adults [8]. In our study over $40 \%$ of those with high heel height wore athletic shoes. Athletic shoes vary significantly with midsole construction that may use elements of gel, foamed polyurethane, or air chambers that serve to aid cushioning [20]. The elevated heel height of athletic shoes may go some way to explain the high heel height observed in this study.

The lack of cushioning found in shoes demonstrates the inadequate amount of structural support for the foot and lower limb. Wear patterns on the footwear provided some indication that they were partially worn and there were considerable amount of medial compression signs. The poor midfoot sole stability and poor heel counter stiffness found suggests that the current footwear does not stabilise the foot during walking. The definition of poor shoes (sandals, slippers and flip-flops) used in this study implies a shoe design with poor fit, poor foot posture, and a lack of shock absorption characteristics. The lack of shock attenuation has the potential to increase loads on plantar tissues, potentially leading to foot pain. Combined with the presence of a flatfoot type, patients with gout wear footwear that gives no support or cushioning and is prone to be unstable. Hence, footwear that has inadequate stability, poor cushioning and limited stability may exacerbate foot pain in patients with gout. 
In the current study the participants reported that fit and comfort were important factors in choosing footwear, suggesting that patients with gout may prioritise these factors due to their condition. More than half of patients reported cost as a factor contributing to their footwear choice. The wearing of poor shoes may be due to financial restrictions when purchasing footwear. Furthermore, gout is a painful and distressing condition that can have a major impact on economically active adults, who may be forced to give up work either temporarily or permanently due to their condition $(23,24)$. These data highlight the barriers related to costs that patients with gout may encounter when purchasing footwear.

We found no relationship between foot length and width with foot pain, disability and impairment. Previous studies have found similar findings in older adults with arthritis (20) and rheumatoid arthritis (10). However, we found significant differences between shoe fit category and foot characteristics with higher scores associated with foot impairment and disability in the poor shoe category. Poor footwear may exacerbate the problem. Lindsay (25) reported that patients with gout may impede the patients' lower limb function, particularly with recreational activities.

In the current study we found high mean BMls indicative of obesity. The findings in the patients with gout are consistent with a previous gout study (7). The sustained repetition of such loading in these activities make significant demands on the feet in normal-weight individuals, and these demands are likely to be magnified in those with gout, obesity and poor footwear. It is possible that the increased demand related to obesity, coupled with the structural changes associated with chronic gout and poor cushioning and control contributes to foot disability in patients with gout.

This study has several limitations. The study was conducted in the summer and in an urban environment, and the results may not be generalisable to other seasons or geographic locations. The footwear questionnaire contains both objective and 
subjective data and not all footwear meet the criteria [8]. For example, open-type footwear such as mules, flip-flops and sandals are difficult to assess and do not have all the footwear features to evaluate. Furthermore, the category for the subjective measure of heel height is based on an arbitrary range (i.e. $0-2.5 \mathrm{~cm} ; 2.6-5.0 \mathrm{~cm}$ and $>5.0 \mathrm{~cm}$ ). Therefore, future research is needed to develop a more objective measure to evaluate of heel height. The current study was cross-sectional and future work using prospective studies is needed to evaluate causative relationships before any definitive conclusions can be made regarding the role of poor footwear in contributing to foot pain, impairment and disability in patients with chronic gout. We did not exclude patients with diabetes from this study. Diabetes is frequently associated with gout [26] and this combination may contribute to more severe foot problems. These patients were not excluded as we wished to ensure that patients with a wide spectrum of disease severity and co-morbidities were included, consistent with gout that is managed in clinical practice. Importantly, sub-analysis of patients with and without diabetes did not show major changes between the groups. Not all patients in this study had microscopically proven gout. The rates of microscopically proven gout are consistent with our previous studies of patients with chronic gout $[27,28]$. Although it is possible that misclassification may have occurred, all patients included in the study had a physician diagnosis of gout and also fulfilled the ACR diagnostic classification for acute gout.

In summary, patients with chronic gout suffer from foot pain, disability and impairment. This study has demonstrated that fit, comfort and costs were perceived by patients to be important factors in choosing footwear although patients current footwear were objectively poor. Overall, the current footwear demonstrated a lack of cushioning, control and stability as well as excessive wear. The majority of shoes worn by patients were also over 12-months old. For patients with gout, this might explain the problems of purchasing adequate footwear due to foot pain, impairment and disability. Based upon the current findings we suggest that footwear should be considered in the management plan of patients with gout. Future research should be focused on assessing the role of competitively priced footwear with adequate cushioning, motion control and sufficient width at the forefoot. 


\section{REFERENCES}

1. Doherty M. New insights into the epidemiology of gout. Rheumatology 2009; 48:ii2-ii8.

2. Perez-Ruiz F. Treating to target: a strategy to cure gout. Rheumatology 2009; 48: ii9-ii14.

3. Roddy E, Zhang W, Doherty M. Are joints affected by gout also affected by osteoarthritis? Ann Rheum Dis 2007; 66:1374-7.

4. Roddy E, Zhang W, Doherty M. Gout and nodal osteoarthritis: a case-control study. Rheumatology 2008; 47: 732-3.Perez F. Treating to target; a strategy to cure gout. Rheumatology 2009; 48: 9-14.

5. Wright SA, Filippucci E, McVeigh C, Grey A, McCarron M, Grassi W et al. Highresolution ultrasonography of the first metatarsophalangeal joint in gout: a controlled study. Ann Rheum Dis 2007; 66:859-864

6. Wang CC, Lien SB, Huang GS, Pan RY, Shen HC,Ko CL et al. Arthroscopic elimination of monosodium urate deposition of the first metatarsophalangeal joint reduces the recurrence of gout. Arthroscopy 2009; 25: 153-158.

7. Rome K, Supervalli D, Sanders A, McQueen FM, McNair P, Dalbeth N. Functional and biomechanical characteristics of foot disease in chronic gout: a case-control study. Clin Biomech 2011; 26: 90-94.

8. Barton CJ, Bonanno D, Menz HB. Development and evaluation of a tool for the assessment of footwear characteristics. J Foot Ankle Res 2009; 2: 10.

9. Otter SJ, Lucas K, Springett K, Moore A, Davies K, Cheek L et al. Foot pain in rheumatoid arthritis prevalence, risk factors and management: an epidemiological study. Clin Rheumatol 2010, 29:255-71.

10.Silvester, R, Rome K, Williams AE, Dalbeth N. 'Choosing shoes'; the challenges for clinicians in assessing rheumatoid footwear: a preliminary study. J Foot Ankle Res 3, 14.

11. Doi T, Yamaguchi R, Asai T, Komatsu $M$ et al. The effect of shoe fit on gait in community-dwelling older adults. Gait Posture [in press].

12. Riskowski J, Dufour AB, Hannan MT. Arthritis, foot pain and shoe wear: current musculoskeletal research on feet. Current Opinion Rheumatol 2011; 23:148-55. 
13. Gurney JK, Kersting UG, Rosenbaum D. Dynamic foot function and morphology in elite rugby league athletes of different ethnicity. Appl Ergon 2009; 40:554-9.

14. Wallace SL, Robinson H, Masi AT. Preliminary criteria for the classification of the acute arthritis of primary gout. Arthritis Rheum 1977; 20: 895-900.

15. Redmond AC, Crane YZ. Menz HB. Normative values for the Foot Posture Index. J Foot Ankle Res 2008, 1:6doi:10.1186/1757-1146-1-6.

16. Helliwell P, Reay N, Gilworth G, Redmond A, Slade A, Tenant A et al. Development of a foot impact scale for rheumatoid arthritis. Arthritis Rheumatism 2005; 53:418-422.

17. Turner DE, Helliwell PS, Siegel KL. Biomechanics of the foot in rheumatoid arthritis: Indentifying abnormal function and the factors associated with localised disease 'impact'. Clin Biomech 2009; 23: 93-100.

18. Budlman-Mak E, Conrad KJ, Roach KE. The Foot Function Index: A measure of foot pain and disability. J Clin Epidemiol 1991; 44: 561-570.

19. Williams AE, Rome K, Nester CJA. Clinical trial of specialist footwear for patients with rheumatoid arthritis. Rheumatology 2007; 46: 302-307.

20.Dufour AB, Broe KE, Nguyen US, Gagnon DR, Hillstrom HJ, Walker AH, et al. Foot pain: is current or past shoewear a factor? Arthritis Rheum 2009; 61:1352-8.

21. Williams AE, Nester CJ. Patient perceptions of stock footwear design features. Prosthet Orthot Int 2006; 30:61-71.

22. Shroyer JF, Weimar WH, Garner J, Knight AC, Sumner AM. Influence of sneakers versus flip-flops on attack angle and peak vertical force at heel contact. Med Sci Sport Exerc 2008; 40: S333.

23. Kleinman NL, Brook RA, Patel PA, Melkonian AK, Brizee TJ, Smeeding JE et al. The impact of gout on work absence and productivity. Value Health 2007; 10:231-7.

24. Brook RA, Forsythe A, Smeeding JE, Edwards NL. Chronic gout: epidemiology, disease progression, treatment and disease burden Current Med Res Opinion 2010; 26: 813-2821

25. Lindsay K, Gow P, Vanderpyl J, Logo P, Dalbeth N. The experience and impact of living with gout: A study of men with chronic gout using a qualitative grounded theory approach. J Clin Rheumatol 2011; 17: 1-6. 
26. Suppiah R, Dissanayake A, Dalbeth N. High prevalence of gout in patients with Type 2 diabetes: male sex, renal impairment, and diuretic use are major risk factors. N Z Med J 2008; 121: 43-50.

27. Dalbeth N, Kumar S, Stamp L, Gow P. Dose adjustment of allopurinol according to creatinine clearance does not provide adequate control of hyperuricemia in patients with gout. J Rheumatol 2006; 33:1646-1650.

28. Dalbeth N, Doyle A, Boyer L, Rome K, Survepalli D, Sanders A et al. Development of a computed tomography (CT) method of scoring bone erosion in the foot and ankle of patients with gout: validation and clinical implications. Rheumatol 2011; 50: 410-416. 


\section{Table 1: Clinical and foot characteristics}

\begin{tabular}{|c|c|}
\hline Age, years, mean (SD) & $63.1(13.2)$ \\
\hline Male sex, $\mathrm{n}(\%)$ & $42(84 \%)$ \\
\hline Ethnicity, n (\%) & Caucasian, 26 (52\%) \\
\hline & Pacific Island, 13 (26\%) \\
\hline$(a)$ & Maori, 8 (16\%) \\
\hline & Asian, 1 (2\%) \\
\hline & Indian, 2 (4\%) \\
\hline Disease duration, years, mean (SD) & $17.9(14.4)$ \\
\hline Cardiovascular disease, $\mathrm{n}(\%)$ & $20(40 \%)$ \\
\hline Diabetes, n (\%) & $7(14 \%)$ \\
\hline Diuretic use, n (\%) & $10(20 \%)$ \\
\hline Allopurinol use, $\mathrm{n}(\%)$ & $28(56 \%)$ \\
\hline Body mass index, $\mathrm{kg} / \mathrm{m}^{2}$, mean (SD) & $31.9(7.8)$ \\
\hline Tophi present, n (\%) & $8(16 \%)$ \\
\hline Serum urate, $\mathrm{mmol} / \mathrm{L}$, mean $(\mathrm{SD})$ & $0.43(0.14)$ \\
\hline Crystal-confirmed diagnosis, $\mathrm{n}(\%)$ & $\underline{18(36 \%)}$ \\
\hline Foot Posture Index, mean (SD) & $4.1(2.9)$ \\
\hline Leeds Foot Impact Scale (impairment), mean (SD) & $8.3(5.3)$ \\
\hline Leeds Foot Impact Scale (activities), mean (SD) & $13.3(10.7)$ \\
\hline Foot Function Index (pain), mean (SD) & $34.0(28.3)$ \\
\hline Foot Function Index (disability), mean (SD) & $33.4(29.1)$ \\
\hline Foot Function Index (activities), mean (SD) & $18.1(24.7)$ \\
\hline
\end{tabular}


Table 2: Footwear Type

\begin{tabular}{|llll|}
\hline Footwear suitability & Total & Footwear type & n (\%) \\
\hline Good & & Oxford Shoe & $9(18 \%)$ \\
& \multirow{2}{*}{$28(56 \%)$} & Walking Shoe & $4(8 \%)$ \\
& & Athletic Shoe & $13(26 \%)$ \\
\hline Average & Therapeutic Footwear & $2(4 \%)$ \\
\hline & $1(2 \%)$ & Boot & $1(2 \%)$ \\
\hline Poor & & Sandal & $6(12 \%)$ \\
& & Flip-flop & $7(14 \%)$ \\
& & Slipper & $4(8 \%)$ \\
& & Backless Slipper & $3(6 \%)$ \\
& & Moccasin & $1(2 \%)$ \\
\hline
\end{tabular}


Table 3: Footwear Construction Characteristics

\begin{tabular}{|ll|}
\hline Footwear Variable & $\mathbf{n}(\%)$ \\
Fit of Shoe & \\
Length & \\
Good & $21(42 \%)$ \\
Too short & $12(24 \%)$ \\
Too long & $17(34 \%)$ \\
Width & \\
Good & $20(40 \%)$ \\
Too narrow & $27(54 \%)$ \\
Too wide & $3(6 \%)$ \\
Depth & \\
Good & $31(62 \%)$ \\
Too shallow & $19(38 \%)$ \\
Heel Height & \\
$0-2.5 \mathrm{~cm}$ & $15(30 \%)$ \\
$2.6-5.0 \mathrm{~cm}$ & $32(64 \%)$ \\
$>5.0 \mathrm{~cm}$ & $3(6 \%)$ \\
Forefoot Height & \\
$0-0.9 \mathrm{~cm}$ & $11(22 \%)$ \\
$1.0-2.0 \mathrm{~cm}$ & $33(66 \%)$ \\
$>2.0 \mathrm{~cm}$ & $6(12 \%)$ \\
Age & \\
$<6$ months & $17(34 \%)$ \\
$6-12$ months \\
$>12$ months & $7(14 \%)$ \\
Width, mm, mean (SD) & $26(52 \%)$ \\
Length, mm, mean (SD) & $23.7(2.44)$ \\
Motion Control Properties & $271.6(18.9)$ \\
1. Midfoot Sole Sagittal Stability & \\
\hline & \\
\hline
\end{tabular}




\begin{tabular}{|c|c|}
\hline Minimal & $25(50 \%)$ \\
\hline Moderate & $16(32 \%)$ \\
\hline Rigid & $9(18 \%)$ \\
\hline \multicolumn{2}{|c|}{ 2. Midfoot Sole Frontal Stability } \\
\hline Minimal & $21(42 \%)$ \\
\hline Moderate & $17(34 \%)$ \\
\hline Rigid & $12(24 \%)$ \\
\hline \multicolumn{2}{|c|}{ 3. Heel Counter Stiffness } \\
\hline Not Available & $13(26 \%)$ \\
\hline Minimal & $21(42 \%)$ \\
\hline Moderate & $3(6 \%)$ \\
\hline Rigid & $13(26 \%)$ \\
\hline \multicolumn{2}{|l|}{ 4. Density } \\
\hline Single & $48(96 \%)$ \\
\hline Dual & $2(4 \%)$ \\
\hline \multicolumn{2}{|l|}{ 5. Fixation } \\
\hline None & $12(23 \%)$ \\
\hline Laces & $21(44 \%)$ \\
\hline Straps/Buckles & $17(33 \%)$ \\
\hline \multicolumn{2}{|c|}{ Presence of Cushioning } \\
\hline None & $30(60 \%)$ \\
\hline Heel & $2(4 \%)$ \\
\hline Heel/forefoot & $18(36 \%)$ \\
\hline \multicolumn{2}{|c|}{ Forefoot Sole Flexion Point } \\
\hline At level of $1^{\text {st }}$ MPJT & $23(46 \%)$ \\
\hline Before $1^{\text {st }}$ MPJT & $27(54 \%)$ \\
\hline \multicolumn{2}{|l|}{ Wear Patterns } \\
\hline \multicolumn{2}{|l|}{ Upper Wear } \\
\hline Medial tilt & $21(42 \%)$ \\
\hline Neutral & $27(54 \%)$ \\
\hline Lateral tilt & $2(4 \%)$ \\
\hline Midsole Wear & \\
\hline
\end{tabular}




\begin{tabular}{|ll|}
\hline Medial compression signs & $10(20 \%)$ \\
Neutral & $37(74 \%)$ \\
Lateral compression signs & $3(6 \%)$ \\
Tread Pattern & \\
Not worn & $14(28 \%)$ \\
Partly worn & $34(68 \%)$ \\
Fully worn & $2(4 \%)$ \\
Outer wear pattern & \\
None & $14(28 \%)$ \\
Normal & $23(46 \%)$ \\
Lateral & $4(8 \%)$ \\
Medial & $9(18 \%)$ \\
\hline
\end{tabular}


Table 4: Factors relating to footwear choice (more than one response was possible using questionnaire)

\begin{tabular}{|ll|}
\hline Factors & $\mathbf{n}(\%)$ \\
Comfort & $47(98 \%)$ \\
Fit & $43(90 \%)$ \\
Support & $43(90 \%)$ \\
Weight & $30(63 \%)$ \\
Cost & $29(60 \%)$ \\
Sole & $22(46 \%)$ \\
Style & $17(36 \%)$ \\
Colour & $16(33 \%)$ \\
\hline
\end{tabular}


Table 5: Differences between shoe fit category and foot characteristics.

\begin{tabular}{|llll|}
\hline & $\begin{array}{l}\text { Poor Shoe } \\
\text { Category } \\
\text { mean (SD) }\end{array}$ & $\begin{array}{l}\text { Good Shoe } \\
\text { Category } \\
\text { mean (SD) }\end{array}$ & $\begin{array}{l}\mathrm{p} \\
\text { value }\end{array}$ \\
\hline $\begin{array}{l}\text { Leeds Foot Impact Scale } \\
\text { (impairment) }\end{array}$ & $9.9(6.5)$ & $6.5(4.5)$ & 0.05 \\
$\begin{array}{l}\text { Leeds Foot Impact Scale } \\
\text { (activities) }\end{array}$ & $17.2(11.3)$ & $8.2(8.7)$ & 0.01 \\
Foot Function Index (pain) & $43.5(33.7)$ & $27.4(22.4)$ & 0.07 \\
Foot Function Index (impairment) & $45.9(29.2)$ & $24.2(26.4)$ & 0.02 \\
Foot Function Index (limitation) & $24.3(25.3)$ & $24.1(26.4)$ & 0.20 \\
\hline
\end{tabular}

